ISSN 2447-9071

doi https://doi.org/10.36414/rbmc.v7i18.96

Contato para correspondência: Hermínio Maurício da Rocha

Sobrinho

E-mail:

herminio.sobrinho@gmail.com

Conflito de interesse: Não

Financiamento: Recursos próprios

Recebido: 17/06/2021

Aprovado: 12/07/2021

\section{O USO DE CANABIDIOL COMO TERAPIA COMPLEMENTAR NO TRANSTORNO DO ESPECTRO AUTISTA}

\section{The use of cannabidiol as complementary therapy in autistic spectrum disorder}

\author{
Rocha Sobrinho $0^{1,2}$ \\ ${ }^{1}$ Pontifícia Universidade Católica de Goiás - PUC Goiás \\ ${ }^{2}$ Universidade Estadual de Goiás - UEG
}

Isabela Castro Pereira', Pedro Henrique Alves Tertuliano', Hermínio Maurício da

\section{Resumo}

Embora não haja evidências claras sobre as causas do Transtorno do Espectro Autista (TEA), sua incidência tem aumentado mundialmente de modo significativo. Atualmente é considerada uma patologia crônica e complexa do neurodesenvolvimento, resultante de disfunção cerebral de etiologia multifatorial desconhecida, o que sempre dificultou a identificação de sua etiologia em cada paciente. Dessa forma, o tratamento priorizado é sintomático e o Canabidiol (CBD) se apresenta como uma terapia complementar que obteve performance positiva no controle do TEA em ensaios clínicos e laboratoriais. O objetivo deste estudo foi descrever o uso do Canabidiol como terapia complementar para os sintomas do TEA. Esta pesquisa se caracteriza como uma revisão bibliográfica narrativa. Ainda que os canabinoides têm apresentado resultados promissores nos efeitos de alguns sintomas comportamentais do TEA, como por exemplo distúrbios do sono, convulsões e hiperatividade, a influência sobre os sintomas essenciais, como a deficiência de comunicação social, interesses restritos e estereotípicos, a etiopatogenia do TEA ainda permanece desconhecida. Embora os canabinoides (Cannabis medicinal) se apresentem promissores no tratamento dos principais sintomas do TEA, recomendações baseadas em evidências são necessárias para garantir a sua segurança e eficácia. Há a necessidade de mais estudos de longo prazo, com amostras homogêneas em termos de idade, uso de medicamentos, nível de funcionamento e presença / ausência de convulsões. Seria de grande importância a escolha de desfechos primários e secundários específicos, com foco no conjunto de sintomas que poderiam se beneficiar do uso de canabinoides.

Palavras-Chave: Transtorno do Espectro Autista; Cannabis; Canabinoides; Canabidiol; Tratamento.

\begin{abstract}
Although there is no clear evidence on the causes of Autistic Spectrum Disorder (ASD), its incidence has increased significantly worldwide. Currently, it is considered a chronic and complex neurodevelopmental pathology, resulting from cerebral dysfunction of unknown multifactorial etiology, which has always made it difficult to identify its etiology in each patient. Thus, the priority treatment is symptomatic and Cannabidiol (CBD) presents itself as a complementary therapy that obtained positive performance in the control of ASD in clinical and laboratory trials. The objective of this study was to describe the use of Cannabidiol as a complementary therapy for the symptoms of ASD. This research is characterized as a narrative bibliographic review. Although cannibinoids have shown promising results in the effects of some behavioral symptoms of ASD, such as sleep disorders, seizures and hyperactivity, the influence on essential symptoms, such as deficiency in social communication, restricted and stereotypical interests, remains unknown. Although medical cannabis appears to show promise in addressing core ASD symptoms,evidence-based recommendations are necessary to ensure safety and effectiveness. There is a need for more long-term studies, with homogeneous samples in terms of age, use of medications, level of functioning and presence / absence of seizures. It would be of great importance to choose specific primary and secondary outcomes, focusing on the set of symptoms that could benefit from the use of cannabinoids.
\end{abstract}

Keywords: Autism spectrum disorder; Cannabis; Cannabinoids; Cannabidiol; Treatment. 


\section{Introdução}

Bleuler em 1911, foi o primeiro a usar a expressão "autismo" para caracterizar a perda do contato com a realidade. No entanto, foi Kanner em 1943 que o definiu, a partir da observação de um grupo de crianças, e constou se tratar de uma inabilidade inata para estabelecer contato afetivo e interpessoal. ${ }^{1}$ O transtorno do espectro autista (TEA), usualmente conhecido como autismo, é um distúrbio de desenvolvimento complexo que é definido do parâmetro comportamental, com inúmeras etiologias e vários graus de severidade. ${ }^{1} \mathrm{O}$ quadro comportamental do TEA é constituído basicamente em quatro manifestações: déficit qualitativo na interação social, déficit na comunicação, padrões de comportamento repetitivos e estereotipados e um repertório restrito de interesses e atividades. ${ }^{2}$

A etiologia do TEA ainda permanece indefinida, por se tratar de um distúrbio complexo e heterogêneo com graus variados de severidade. ${ }^{3}$ Um estudo aponta que algumas regiões como a amígdala, os gânglios da base e outras regiões do cérebro como o cerebelo, hipocampo e corpo caloso, podem estar envolvidas no desenvolvimento dos sintomas dessa doença. Entretanto, as deficiências celulares e metabólicas, que são base para o desenvolvimento cerebral anormal, ainda permanecem desconhecidas. ${ }^{4}$

O diagnóstico do TEA permanece clínico, sendo que os critérios atualmente utilizados são aqueles descritos no Manual Estatístico e Diagnóstico da Associação Americana de Psiquiatria (DSM-V). Esses critérios têm evoluído com o passar dos anos, e na prática o TEA tem sido usado como categoria diagnóstica em indivíduos com déficits na interação social, déficits em linguagem/comunicação e padrões repetitivos do comportamento. ${ }^{1,5}$

Apesar da psicopatologia exigir tratamento farmacológico, não há medicamentos que tratam os principais sintomas do TEA. A terapia comportamental continua sendo a base do tratamento para sintomas de déficits de comunicação, déficits de interação social e comportamento repetitivo. Atualmente, apenas os medicamentos risperidona e aripiprazol são aprovados pela Food and Drug Administration (FDA) para o tratamento de qualquer comportamento associado ao TEA, entre eles distúrbios de irritabilidade marcados por agressão e comportamento auto prejudicial. ${ }^{6}$

A planta de Cannabis enriquecida com canabidiol (CBD) tem se destacado entre os novos tratamentos complementares para crianças com autismo, visto que tem resultado em muitos benefícios. ${ }^{7}$ Preparações medicinais de flores e resina de Cannabis sativa têm sido usadas na China desde 2700 a.C. para tratar distúrbios menstruais, gota, reumatismo, malária, prisão de ventre e distração. Mais recentemente, a Cannabis tem sido usada para tratar glaucoma, dor, náuseas e vômitos, espasmos musculares, insônia, ansiedade e epilepsia. ${ }^{8,9}$

O CBD pode melhorar vários sintomas do TEA, mesmo em pacientes não epilépticos, com aumento substancial na qualidade de vida tanto para pacientes com TEA quanto para cuidadores. $^{10}$

A literatura ainda é bastante escassa em estudos clínicos abordando o uso da Cannabis medicinal enriquecida com Canabidiol (CBD) como terapia complementar para o tratamento do TEA. Diante do exposto, o presente trabalho apresenta como objetivo descrever o uso do CBD como terapia complementar para os principais sintomas do Transtorno do Espectro Autista (TEA).

\section{Métodos}

Trata-se de uma revisão bibliográfica narrativa. Utilizou-se as bases de dados eletrônicas PubMed (US National Library of Medice), Biblioteca Virtual em Saúde (BVS) e Portal de Periódicos da Capes. Para a busca de artigos nas bases de dados virtuais foram utilizados descritores ou palavras-chave em língua portuguesa e inglesa, especialmente os Descritores em Ciências da Saúde (DeCS): "Autism Spectrum Disorder, Pathogenesis, Complementary Treatment, Cannabidiol, Cannabis". Foram avaliados e selecionados artigos publicados no período do ano 2000 ao ano de 2021. O foco principal desse estudo foi a abordagem do tratamento complementar do TEA com Canabidiol (CBD). Do material pesquisado e encontrado, foram selecionadas as referências encontradas dentro do recorte temporal determinado, os artigos com textos completos, os não duplicados, artigos que apresentaram conteúdos que contribuíram para o cumprimento dos objetivos e da relevância do estudo. Nessa busca, foram excluídos da pesquisa os trabalhos que foram publicados antes do ano de 2000, os duplicados, os não disponíveis com texto completo nas bases de dados utilizadas, bem como aqueles que apresentaram conteúdos que não estavam relacionados aos objetivos desta revisão.

\section{Revisão da literatura sobre os aspectos gerais do Transtorno do Espectro Autista (TEA)}

Embora não haja evidências claras sobre as causas do TEA, sua incidência tem aumentado mundialmente de modo significativo. ${ }^{11} \mathrm{O}$ estudo dessa doença na infância é especial, visto que é principalmente nessa fase em que o diagnóstico é feito, além de que, quanto antes iniciado o tratamento, melhor o prognóstico.

Os sintomas do TEA costumam ser reconhecidos durante o segundo ano de vida, embora já possam ser percebidos antes dos 12 meses de idade, como em casos em que há graves 


\section{Busca nas Bases de Dados \\ PubMed e Periódicos da Capes e Biblioteca Virtual em Saúde (BVS)}

\section{Combinação entre os DeCS e palavras-chave (período de 2000 a 2021):}

Autism Spectrum Disorder, Pathogenesis, Complementary Treatment, Cannabidiol, Cannabis.

Manuscritos encontrados $\mathrm{N}=56$

\begin{tabular}{|c|}
\hline \multicolumn{1}{|c|}{$\begin{array}{c}\text { Seleção Inicial de publicações com } \\
\text { temática relacionada ao estudo } \\
\text { (Screening inicial) } \\
\text { (N=48) }\end{array}$} \\
\hline \multicolumn{1}{|c|}{ Exclusões Diversas } \\
(N=7) \\
$\checkmark$ Duplicadas ( $\mathrm{n}=1$ ) \\
$\checkmark$ Estudos alheios aos objetivos da pesquisa ( $\mathrm{n}=3)$ \\
\hline Artigos incluídos nesta revisão ( $\mathbf{N}=\mathbf{4 1 )}$ \\
\hline
\end{tabular}

Figura 1. Algoritmo adotado para a busca e seleção de artigos na elaboração desta revisão.

atrasos de desenvolvimento. Inicialmente esses sintomas são identificados devido aos atrasos precoces do desenvolvimento ou perdas de habilidades sociais ou linguísticas. ${ }^{12}$

Em geral, aqueles de prejuízo menores podem ser mais capazes de manter sua independência ao exercerem atividades como trabalho na fase adulta; esses tendem a ter linguagem e capacidades intelectuais superiores, conseguindo encontrar um ambiente que combine com seus interesses e habilidades especiais. No entanto, esses adultos são mais propensos a ansiedade, depressão e estresse devido aos esforços de terem que manter características socialmente aceitáveis para a inserção social. Em relação a fase da velhice, ainda existem poucos estudos sobre os comportamentos e cotidiano do idoso com TEA. ${ }^{12}$

\section{Epidemiologia}

O TEA é mais prevalente em homens e frequentemente acompanhado por comorbidades, como distúrbios do sono e déficit de atenção / TDAH. ${ }^{13}$ Nos últimos 10 anos, devido a compreensão das mudanças genéticas e o envolvimento de mutações em expansão no TEA, dezenas de genes foram identificados, respondendo coletivamente por $10-20 \%$ dos casos de TEA. Nos Estados Unidos da América, por exemplo, de 1 para cada 150 crianças de 8 anos em 2000 e 2002, a prevalência do TEA aumentou de 1 para cada 68 crianças em 2010 e 2012, chegando à prevalência de 1 para cada 58 em 2014, mais que duplicando o número de casos durante esse período. Ainda não está claro se taxas mais altas refletem expansão dos critérios diagnósticos do DSM-V de modo a incluir casos subdiagnosticados, maior conscientização, diferenças na metodologia dos estudos ou aumento real na frequência do transtorno. ${ }^{14}$

\section{Manifestações Clínicas}

O autismo é atualmente considerado uma patologia crônica e complexa do neurodesenvolvimento, resultante de disfunção cerebral de etiologia multifatorial desconhecida, com isso cerca de $80 \%$ dos casos ficam sem diagnóstico definitivo. ${ }^{15}$ Manifestações do TEA também variam muito dependendo da gravidade da condição autista, do nível de desenvolvimento e da idade cronológica; daí o uso do termo espectro. Nesse sentido, o TEA pode apresentar uma série de manifestações clínicas como déficits persistentes na comunicação social e na interação social em múltiplos contextos, além de padrões restritos e repetitivos de comportamento, interesses ou

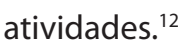

As manifestações devem estar presentes precocemente no período do desenvolvimento e devem causar prejuízo clinicamente significativo no funcionamento social, profissional ou em outras áreas importantes da vida do indivíduo. Déficits na reciprocidade socioemocional e para desenvolver, manter e compreender as relações são particularmente evidentes em crianças pequenas, pois nelas costumam não só existir uma falta de jogo social e imaginação compartilhado, mas também podem apresentar pequena ou nenhuma capacidade de iniciar interações sociais e de compartilhar emoções. ${ }^{12}$

Os sintomas costumam ser reconhecidos durante o segundo ano de vida (12 a 24 meses) de forma que as características comportamentais do TEA tornam-se evidentes na primeira infância, com alguns casos apresentando falta de interesse em interações sociais no primeiro ano de vida, platôs ou regressão no desenvolvimento, com uma deterioração gradual ou relativamente rápida em comportamentos sociais ou uso da linguagem, frequentemente durante os dois primeiros anos de vida. Tais perdas são raras em outros transtornos, podendo ser um sinal de alerta útil para o TEA, além disso, não é um transtorno degenerativo, sendo comum que aprendizagem e compreensão continuem ao longo da vida. ${ }^{12}$ 


\section{Etiopatogenia}

O TEA é um distúrbio complexo e geneticamente heterogêneo, o que sempre dificultou a identificação de sua etiologia em cada paciente. ${ }^{16}$

Embora acredite que fatores ambientais, como infecções ou o uso de medicamentos durante a gestação, tenham papel no desenvolvimento do transtorno, estima-se que o TEA seja hereditário em cerca de 50 a $90 \%$ dos casos, o que demonstra a importância dos fatores genéticos na patogênese da doença. ${ }^{17}$

Pesquisas sobre herança familiar sugeriram genes potenciais alvo, incluindo aqueles relacionados aos receptores de neurotransmissores (serotonina e ácido gama-aminobutírico [GABA]) e controle estrutural do SNC (genes HOX). ${ }^{18}$

As diferenças na estrutura e função cerebrais provavelmente formam a base da etiologia dos transtornos do espectro autista. Algumas crianças com transtornos do espectro do autismo têm aumento dos ventrículos, algumas apresentam hipoplasia do vérmis cerebelar e outras têm anormalidades dos núcleos do tronco cerebral. ${ }^{19}$

Há também algumas síndromes genéticas que envolvem mutações nos epígenos associados com risco aumentado para TEA como síndrome de Rett, síndrome do X frágil, síndrome de Di George, Turner, Angelman, entre outras. ${ }^{19}$ Os principais fatores relacionados à etiopatogenia do TEA estão resumidos na figura 2.

\section{Tratamento Farmacológico}

O tratamento farmacológico é responsável por controlar as desordens comportamentais, e assim melhorar a qualidade de vida e promover o convívio social dos pacientes, no entanto, não possui ação direta nas causas do TEA. ${ }^{20} \mathrm{O}$ tratamento farmacológico tem o intuito de controlar sinais específicos do TEA, como agressividade e/ou comportamentos estereotipados, entendidos como "indesejados", permitindo a abordagem de outras terapias. Além disso, por não existir um tratamento específico, muitas vezes é necessário fazer as intervenções farmacológicas para atenuar esses sintomas.

Os antipsicóticos, em especial, a risperidona, são bastante utilizados no tratamento dos sintomas globais do autismo. Estudos comprovam uma melhora nos sintomas dos comportamentos restritivos, repetitivos e estereotipados (CRRE) como agressividade, irritabilidade e estresse. Salientando que essa intervenção só deverá ser empregada, juntamente com outras terapias e quando o indivíduo com TEA não estiver apresentando sucesso nas respostas. ${ }^{21}$

Sabe-se que os inibidores seletivos da recaptação de serotonina, como a fluoxetina e sertralina entre outros, são utilizados no tratamento do TEA, com o objetivo de reduzir
Figura 2. Fluxograma sintetizando a etiopatogenia do Transtorno do Espectro Autista.

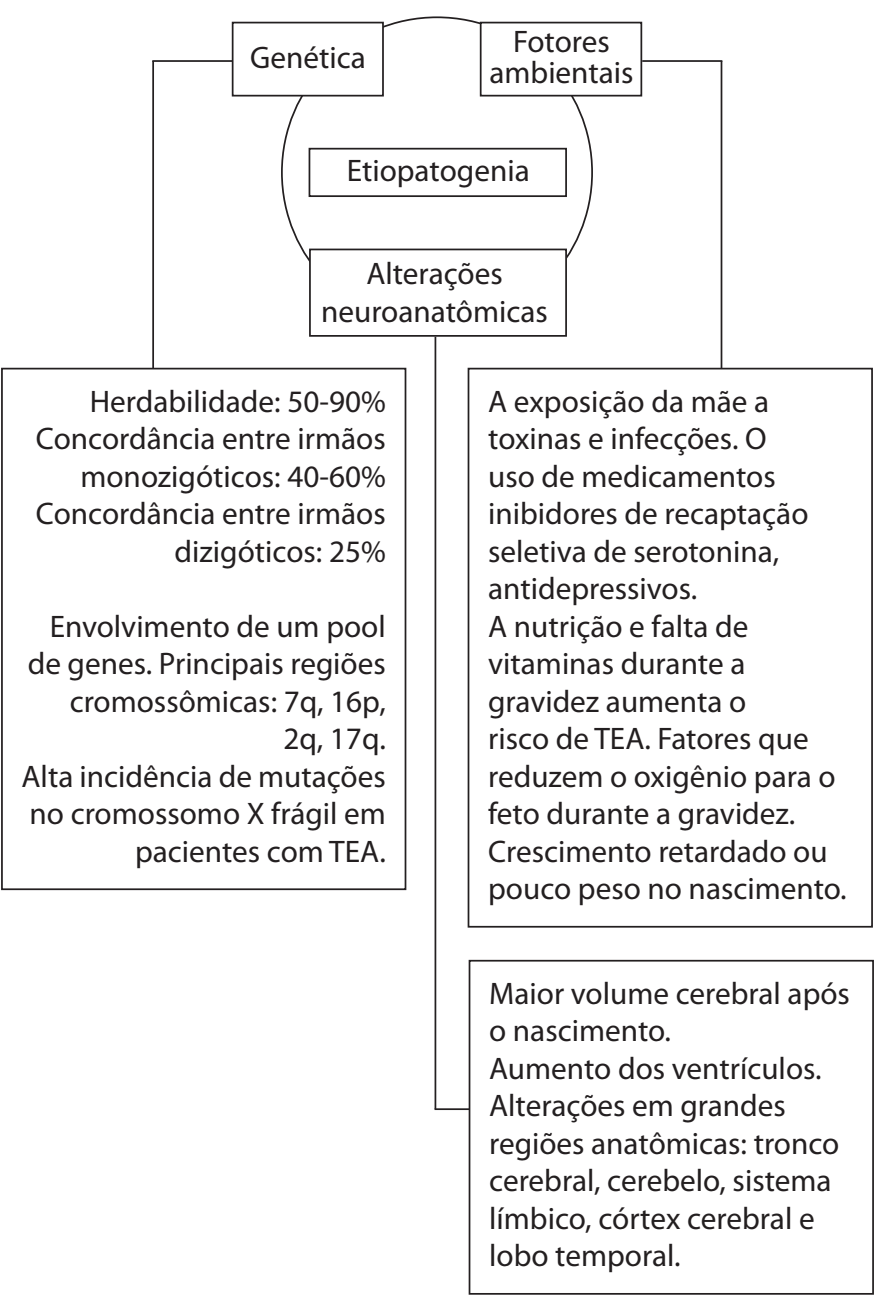

Fonte: Adaptado de Gyawali e Patra, 2019 (18).

os comportamentos obsessivos, rituais e estereotipias, apresentando eficácia variável. Os estabilizadores de humor, como o divalproato de sódio, têm como indicação principal o tratamento do Transtorno Bipolar. Entretanto, estudos sobre o uso desse medicamento no tratamento de crianças autistas demonstram que alterações favoráveis na instabilidade, comportamentos repetitivos e agressão são presentes, além disso a polifarmácia torna os pacientes vulneráveis às interações medicamentosas e sérios efeitos adversos. ${ }^{18,22}$

\section{Terapia Complementar - Canabidiol (CBD)}

A planta de cannabis contém centenas de produtos químicos diferentes, com mais de 100 componentes, conhecidos como canabinoides. ${ }^{23}$ Os canabinoides são frequentemente divididos em três subgrupos: fitocanabinoides, 
endocanabinoides e canabinoides sintéticos. Os fitocanabinoides são os canabinóides naturais da planta e desses, o Canabidiol (CBD) é um constituinte não psicotrópico da planta Cannabis, o segundo mais abundante. ${ }^{24} \mathrm{O} C B D$ exibe uma infinidade de propriedades farmacológicas, incluindo anticonvulsivo, sedativo, hipnótico, antipsicótico, anti-inflamatório e neuroprotetor. O CBD também exibe efeitos antioxidantes e promove neurogênese e parece ser bem tolerada pelos seres humanos. ${ }^{25,26} \mathrm{O}$ sistema endocanabinoide atua como uma rede neuromoduladora envolvida na regulação das respostas emocionais, reatividade comportamental ao contexto e interação social. ${ }^{27}$

Atualmente, não existem tratamentos específicos disponíveis para o TEA, assim as intervenções terapêuticas são mais focadas na diminuição dos comportamentos disruptivos, treinamento e ensino de habilidades de autoajuda para uma maior independência do paciente. ${ }^{28}$ Nesse sentido, existe um interesse crescente em canabinoides, especialmente CBD, como tratamento complementar para não só os sintomas e comorbidades do TEA, mas também para manutenção adequada do funcionamento social. ${ }^{29}$

O CBD também pode potencializar alguns dos efeitos benéficos do $\triangle$ 9-THC, pois reduz a psicoatividade do $\Delta$ 9-THC para aumentar sua tolerabilidade e ampliar sua janela terapêutica. $O$ CBD pode neutralizar algumas das consequências funcionais da ativação do CB1 no cérebro. ${ }^{30}$ Possivelmente pelo aumento indireto da atividade dos receptores da adenosina. Isso demonstra em parte por que usuários de preparações de Cannabis com altas taxas de CBD: $\triangle$ 9-THC têm menos probabilidade de desenvolver sintomas psicóticos do que aqueles que consomem preparações com baixas concentrações de CBD: $\triangle 9-$ THC. $^{31}$

Além do CBD outros canabinoides tem se mostrado benéfico em modelos experimentais de vários distúrbios neurológicos, incluindo convulsão e epilepsia, como o canabicromeno, o $\Delta 9$-tetrahidrocanabivarina ( $\triangle 9-\mathrm{THCV}$ ) e canabidivarina (CBDV). Esses outros canabinoides, assim como o CBD, atuam de forma positiva no tratamento de doença de Parkinson, estão envolvidos na redução da neuroinflamação e produzem efeitos anticonvulsivantes. ${ }^{32}$

Um estudo recente evidenciou que o CBD tem uma influência positiva no comportamento social de maneira que surtos comportamentais foram melhorados em $61 \%$ dos pacientes portadores de TEA, problemas de comunicação em 47\%, ansiedade em $39 \%$, estresse em $33 \%$ e comportamento perturbador em $33 \%$ dos pacientes. Essa situação acontece pois o tratamento baseia-se nas observações e na teoria de que os efeitos do CBD podem incluir o alívio da psicose, ansiedade, facilitação do sono REM e atua suprimindo a atividade convulsiva. ${ }^{33}$ Outro estudo demonstrou também significativa melhora na qualidade de vida nas famílias de pacientes com TEA sendo relatados maior autonomia dos pacientes com atividades simples como tomar banho e trocar de roupa, além disso, houve significativa melhora no humor, diminuição das convulsões e dos ataques de raiva. ${ }^{34}$

Em um estudo prospectivo foi observado que no tratamento de crianças com extrato vegetal que continha CBD e THC na proporção de 20:1, houve melhora geral no comportamento, ansiedade e comunicação avaliada pelos pais na escala Clinical Global Impression of Change (CGIC). Melhoria considerável nos problemas de comportamento foi relatada em $61 \%$ das crianças. Melhora considerável nos problemas de ansiedade e comunicação foi relatada em $39 \%$ e $47 \%$ das crianças. Além disso, após o tratamento com CBD, 33\% receberam menos medicamentos concomitantes ou dosagem mais baixa, 24\% pararam de tomar os medicamentos. Entretanto, $8 \%$ receberam mais medicamentos ou dose mais alta e $5 \%$ interromperam o tratamento devido a efeitos colaterais que incluíram irritabilidade acentuada após o início do tratamento.? Esses dados pré-clínicos e os resultados do estudo demonstram que vale a pena uma exploração mais aprofundada do tratamento com CBD em estudos controlados. Até que tenha mais evidências disponíveis, os médicos devem ser cautelosos no uso de CBD medicinal em crianças com TEA.

\section{Mecanismos de ação do Canabidiol (CBD)}

O sistema endocanabinoide (SEC) é constituído por uma série de receptores específicos sendo o $\mathrm{CB} 1$ e $\mathrm{CB} 2$ os principais. São ativados por ligantes (ex.: Canabidiol-CBD) envolvendo uma variedade de enzimas, transportadores e outras proteínas relacionadas à síntese, mobilização e captação destas micromoléculas. Dentre estas enzimas, se encontram as relacionadas à biossíntese e degradação dos endocanabinoides, tais como Amida Hidrolase de Ácidos Graxos, do inglês, Fatty Acid Amide Hydrolase (FAAH) e lipase de monoacilglicerol (MAGL). A ativação dos receptores canabinoides afeta as ações de vários neurotransmissores como a acetilcolina (ACh), dopamina, GABA, glutamato, serotonina, noradrenalina e opioides endógenos. ${ }^{29}$

Outros receptores também podem ser modulados pelo $\mathrm{CBD}$, incluindo receptores de potencial transiente vaniloides (ex. TRPV1, TRPV2, TRPA1) onde atua como agonista, sendo antagonista de receptor de potencial transiente melastatina tipo 8 (TRP), receptor órfão ligado à proteína G (GPR55), receptor de potencial transiente de proteínas transmembranas ancrinas (TRPA), receptor serotoninérgico (5-HT1A) e PPAR $\gamma$, 
inibindo da recaptação sináptica de noradrenalina, GABA, adenosina, bem como de dopamina e estimulando a atividade de receptores de glicina $\alpha 3$ e $\alpha 1 .^{35}$

Os endocanabinoides não são armazenados em vesículas, sendo imediatamente liberados após a ativação pos-sináptica para atuarem na modulação dos neurônios pré-sinápticos, processo este denominado neurotransmissão retrógrada. Atuam "sob demanda": são acionados quando necessário e funcionam para reparar ou modular a função de outros mediadores. Sua ação é encerrada com a captação nas terminações pré-sinápticas. Os receptores canabinoides se encontram inseridos na membrana celular, acoplados às proteínas-G e à enzima adenilato ciclase (AC), e participam do processo de transdução de sinais. $O$ aumento do cálcio intracelular é fator desencadeante para que o precursor de endocanabinoide acoplado à membrana seja sintetizado, clivado e liberado. Após essa interação, há reações em vários componentes intercelulares, que incluem a inibição da AC, abertura dos canais de potássio, diminuindo a transmissão dos sinais e fechamento dos canais de cálcio voltagem-dependentes, levando a um decréscimo na liberação de neurotransmissores. Com isso, ocorre diminuição da liberação de neurotransmissores pelo neurônio pré-sináptico. ${ }^{35,36} \mathrm{Os}$ possíveis alvos moleculares do Canabidiol (CBD) e mecanismos associados aos seus efeitos neuroprotetores são apresentados, a seguir, na figura 3 .

Figura 3. Possíveis alvos moleculares do Canabidiol (CBD) e mecanismos associados aos seus efeitos neuroprotetores. Fonte: Adaptado de Franco e Viegas, $2017^{(35)}$.

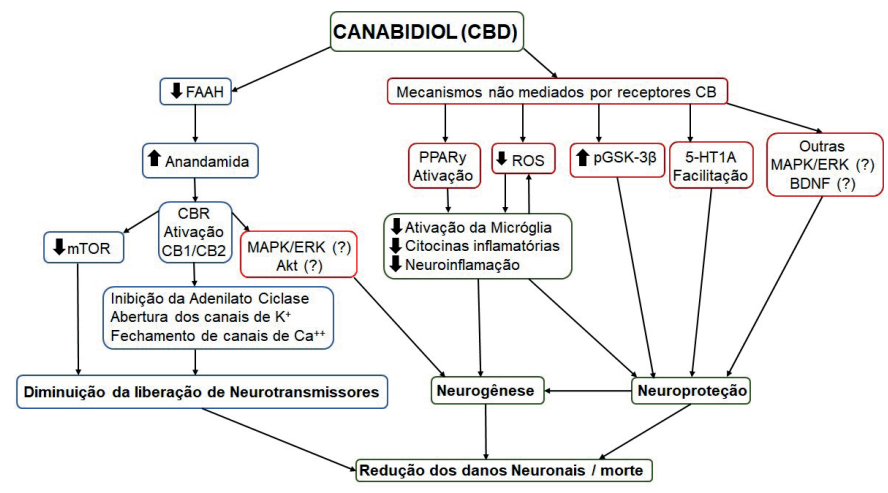

Legenda: $m T O R=$ Alvo mecanístico da rapamicina; $F A A H=$ Amida hidrolase de ácidos graxos; MAGL=Lipase de monoacilglicerol; $C B R=$ Receptores canabinoides; MAPK/ERK=Via de sinalização da cinase regulada por fatores extracelulares, da família das proteínas cinases ativadas por mitógenos; Akt=Proteína quinase $B$; PPARy=Receptores ativados por proliferadores de

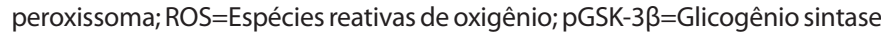
quinase $3 \beta ; 5-\mathrm{HT}-1 \mathrm{~A}=$ Receptores de serotonina; BNDF=Fator Neurotrófico derivado do cérebro.

\section{Resultados e Discussão}

Alguns estudos clínicos avaliaram o desempenho da
Cannabis no tratamento complementar do TEA. Um estudo clínico não controlado avaliou o uso de dronabinol (delta-9THC) em uma criança com TEA sem associação com outros medicamentos, sem efeitos adversos, apresentou melhora no comportamento com diminuição da irritabilidade, da hiperatividade e letargia. ${ }^{37}$ Um relato de caso que avaliou o uso do extrato de canabidiol teve resultados semelhantes ao estudo anterior. ${ }^{38}$

Três estudos prospectivos avaliaram o desempenho de extrato de canabidiol sendo um deles na proporção 75:1 de CBD/THC e os outros dois na proporção 20:1 de CBD/THC em solução oleosa. No estudo de Fleury-Teixeira e colaboradores (2019) foram avaliados 18 crianças e adolescentes, desses, 3 abandonaram o tratamento devido efeitos adversos que podem ter acontecido em função da interação do canabidiol com medicamentos psiquiátricos usados concomitantemente, 15 pacientes apresentaram melhora dos sintomas comportamentais como distúrbios no sono, convulsões, e crises comportamentais, além disso foram observados sinais de melhora no desenvolvimento motor, no comportamento social, comunicação e performance cognitiva. Dentre os 15 pacientes que continuaram o tratamento apenas 1, que fazia associação de vários medicamentos psicotrópicos, abandou o tratamento no meio do estudo devido piora dos sintomas, o restante manteve os resultados. ${ }^{10}$ No estudo de Bar-Lev Schleider e colaboradores (2019), a amostra foi de 188 crianças e adolescentes que apresentavam TEA, epilepsia e TDHA. Com um mês de tratamento 179 pacientes mantiveram o tratamento ativo demonstrando melhora significativa dos sintomas comportamentais com exceção de 7 que apresentaram efeitos adversos como sonolência, refluxo e falta de apetite. Após 6 meses 155 pacientes mantiveram o tratamento e 15 pararam, 12 por não apresentarem diferença no tratamento e os outros 3 por causa dos efeitos adversos. ${ }^{34}$ No estudo de Barchel e colaboradores (2019) foram avaliados 53 crianças e adolescentes com TEA que demonstraram melhoria na hiperatividade $(68,4 \%)$, diminuição de auto lesão $(67,6 \%)$, melhoria nos problemas de sono $(71,4 \%)$, ansiedade $(47,1 \%)$ e melhoria geral em $74,5 \%$, entretanto alguns pacientes tiveram piora na sonolência, diminuição de apetite e insônia. ${ }^{39}$

Também foram avaliados outros três estudos retrospectivos. No estudo de Aran e colaboradores (2019)in 60 children with ASD and severe behavioral problems (age $=11.8 \pm 3.5$, range $5.0-17.5 ; 77 \%$ low functioning; $83 \%$ boys 60 crianças com TEA e problemas comportamentais foram tratadas com óleo canabinoide de CBC e THC na proporção de 20:1 e apresentaram melhora no comportamento e estresse em $62 \%$, ansiedade melhorou em $39 \%$ e comunicação em $47 \%$, 
além disso, 14\% apresentaram distúrbio do sono, inquietação, nervosismo e perda de apetite em 9\%.7 No estudo de Kuester e colaboradores (2017) 20 pacientes com TEA foram tratados com extrato de CBD e THC equilibrado em 19\% e $9,5 \%$, demonstrou que $66,7 \%$ tiveram melhora significativa no estresse e comportamento e a maioria dos casos melhorou pelo menos um dos principais sintomas do transtorno, porém dois pacientes apresentaram mais agitação e um teve mais irritabilidade..$^{40}$ No estudo de McVige e colaboradores (2020) self-injurious behavior (SIB foram avaliados 20 pacientes com TEA que fizeram uso de Cannabis medicinal observou-se uma melhora em todas as áreas comportamentais, na frequência e gravidade das crises, no grau de dor geral e melhora do sono, porém três pacientes apresentaram efeitos adversos leves não especificados. ${ }^{41}$

Dessa forma, todos os estudos analisados apresentaram resultados favoráveis e demostraram melhora nos sintomas comportamentais dos pacientes com TEA. Com isso as evidências encontradas demonstraram que os canabinoides podem exercer efeitos benéficos e podem diminuir a necessidade do uso de medicamentos psiquiátricos. ${ }^{34,37,38}$ Observou-se que os principais efeitos adversos foram sonolência, aumento do apetite e irritabilidade, mas muitos pacientes fizeram o tratamento concomitante com medicamentos psiquiátricos, por isso é difícil definir se os efeitos adversos foram causados pelos extratos canabinoides ou por outras drogas. ${ }^{10,39-41}$ Além disso os trabalhos são bastante mistos sendo que alguns incluíram crianças e adolescentes, outros crianças com TEA e TDAH. Um breve resumo destes estudos é apresentado na tabela 1, a seguir.

\section{Conclusão}

A etiopatogênese do TEA ainda permanece amplamente obscura. Entre as hipóteses biológicas, estudos evidenciaram um desequilíbrio no sistema endocanabinoide (SCB), que regula algumas funções tipicamente prejudicadas no TEA, como respostas emocionais e interação social. O canabidiol (CBD) é o componente não intoxicante da Cannabis sativa que pode melhorar sintomas relacionados ao TEA, além de proporcionar efeitos colaterais que quando comparados com os de tratamentos farmacológicos podem ser considerados menos agressivos.

Ainda que os canibinoides apresentaram resultados promissores nos efeitos de alguns sintomas comportamentais do TEA, como por exemplo distúrbios do sono, convulsões e hiperatividade, a influência sobre os sintomas essenciais, como a deficiência de comunicação social, interesses restritos e estereotípicos, a etiopatogenia desta doença ainda permanece desconhecida.

Ainda que alguns ensaios clínicos estejam em andamento, ainda há a necessidade de mais estudos de longo prazo, com amostras homogêneas em termos de idade, uso de medicamentos, nível de funcionamento e presença / ausência de convulsões. Seria de grande importância a escolha de desfechos primários e secundários específicos, com foco no conjunto de sintomas que poderiam se beneficiar do uso de canabinoides.

Embora os canabinoides (Cannabis medicinal) se apresentem promissores no tratamento dos principais sintomas do TEA, recomendações baseadas em evidências são necessárias para garantir a sua segurança e eficácia.

\section{Referências bibliográficas}

1. Gadia CA, Tuchman R, Rotta NT. Autismo e doenças invasivas de desenvolvimento. J Pediatr (Rio J). 2004;80(2):83-94.

2. Pereira AM, Riesgo RS, Wagner MB. Childhood autism: Translation and validation of the Childhood Autism Rating Scale for use in Brazil. J Pediatr (Rio J). 2008;84(6):487-94.

3. Ozand PT, Al-odaib A, Merza H, Harbi S Al. Autism : a review. 2003;1(2):55-67.

4. Dawson G, Webb S, Schellenberg GD, Dager S, Friedman S, Aylward E, et al. Defining the broader phenotype of autism: genetic, brain, and behavioral perspectives. Dev Psychopathol. 2002;14(3):581-611.

5. Rutter M. Incidence of autism spectrum disorders: Changes over time and their meaning. Acta Paediatr Int J Paediatr. 2005;94(1):2-15.

6. Lamy M, Erickson CA. Pharmacological management of behavioral disturbances in children and adolescents with autism spectrum disorders. Curr Probl Pediatr Adolesc Health Care [Internet]. 2018;48(10):250-64.

7. Aran A, Cassuto H, Lubotzky A, Wattad N, Hazan E. Brief Report: Cannabidiol-Rich Cannabis in Children with Autism Spectrum Disorder and Severe Behavioral Problems-A Retrospective Feasibility Study. J Autism Dev Disord [Internet]. 2019;49(3):1284-8.

8. Flachenecker P. A new multiple sclerosis spasticity treatment option: effect in everyday clinical practice and cost-effectiveness in Germany. Expert Rev Neurother. 2013 Feb;13(3 Suppl 1):15-9.

9. Machado Rocha FC, Stéfano SC, De Cássia Haiek R, Rosa Oliveira LMQ, Da Silveira DX. Therapeutic use of Cannabis sativa on chemotherapy-induced nausea and vomiting among cancer patients: systematic review and meta-analysis. Eur J Cancer Care (Engl). 2008 Sep;17(5):431-43. 
Tabela 1 - Estudos que avaliaram o desempenho de produtos da canabis no tratamento complementar do Transtorno do Espectro Autista.

\begin{tabular}{|c|c|c|c|c|c|}
\hline $\begin{array}{l}\text { Tamanho da } \\
\text { amostra (n) }\end{array}$ & $\begin{array}{l}\text { População/ } \\
\text { País }\end{array}$ & Tipo de estudo & Substância usada & Resultados & Referência \\
\hline$N=1$ & $\begin{array}{l}\text { Criança com TEA } \\
+ \text { PGC } \\
\text { (Áustria) }\end{array}$ & Relato de caso & $\begin{array}{l}\text { Dronabidiol }(\Delta 9- \\
T H C)\end{array}$ & $\begin{array}{l}\text { Melhora dos sintomas do } \\
\text { autismo, hiperatividade, } \\
\text { letargia e irritabilidade. }\end{array}$ & (37) \\
\hline $\mathrm{N}=18$ & $\begin{array}{l}\text { Crianças } \\
\text { adolescentes } \\
17 \text { anos) com TEA } \\
\text { (Brasil) }\end{array}$ & $\begin{array}{l}\text { Estudo de coorte } \\
\text { prospectivo }\end{array}$ & $\begin{array}{l}\text { Extrato de CBD } \\
\text { enriquecido } 75: 1 \\
\text { proporção CBD para } \\
\text { THC }\end{array}$ & $\begin{array}{l}\text { Melhora na maioria } \\
\text { dos } \\
\text { comportamentais. }\end{array}$ & $\begin{array}{l}\text { (10)motor skills, social interaction, } \\
\text { communication, and behavior. This set } \\
\text { of functional deficits often results in } \\
\text { lack of independence for the diagnosed } \\
\text { individuals, and severe distress for } \\
\text { patients, families, and caregivers. There is } \\
\text { a mounting body of evidence indicating } \\
\text { the effectiveness of pure cannabidiol }\end{array}$ \\
\hline
\end{tabular}

\begin{tabular}{|c|c|c|c|c|c|}
\hline$N=53$ & $\begin{array}{l}\text { Crianças } \\
\text { adolescentes } \\
22 \text { anos) com TEA } \\
\text { e PGC } \\
\text { (Israel) }\end{array}$ & $\begin{array}{l}\text { Estudo de coorte } \\
\text { prospectivo }\end{array}$ & 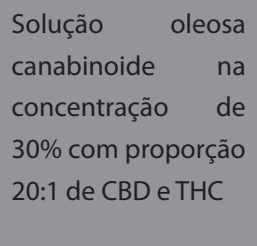 & $\begin{array}{l}\text { Melhora na agressividade, } \\
\text { hiperatividade e } \\
\text { ansiedade. }\end{array}$ & (39) \\
\hline$N=60$ & $\begin{array}{l}\text { Crianças com TEA } \\
\text { e PGC } \\
\text { (Israel) }\end{array}$ & $\begin{array}{l}\text { Estudo de coorte } \\
\text { retrospectivo }\end{array}$ & $\begin{array}{l}\text { CBD e THC na } \\
\text { proporção } \quad 20: 1 \\
\text { dissolvido em óleo } \\
\text { de oliva }\end{array}$ & $\begin{array}{l}\text { Melhora considerável } \\
\text { nos sintomas } \\
\text { comportamentais. }\end{array}$ & $\begin{array}{c}\text { (7)in } 60 \text { children with ASD and severe } \\
\text { behavioral problems (age }=11.8 \pm 3.5 \\
\text { range } 5.0-17.5 ; 77 \% \text { low functioning; } 83 \% \\
\text { boys }\end{array}$ \\
\hline$N=188$ & $\begin{array}{l}\text { Crianças e } \\
\text { adolescentes 12,9 } \\
\text { - } 7 \text { anos com TEA, } \\
\text { epilepsia e TDAH } \\
\text { (Israel) }\end{array}$ & $\begin{array}{l}\text { Estudo de coorte } \\
\text { prospectivo }\end{array}$ & $\begin{array}{l}\text { Óleo na proporção } \\
\text { 20:1 de CBD e THC }\end{array}$ & $\begin{array}{l}\text { Grande } \begin{array}{l}\text { melhora } \\
\text { nos }\end{array} \text { comas } \\
\text { com porta m e n t a is, } \\
\text { agitação e agressividade. }\end{array}$ & (34) \\
\hline$N=20$ & $\begin{array}{l}\text { Crianças com TEA } \\
\text { (Egito) }\end{array}$ & $\begin{array}{l}\mathrm{E} \mathrm{s} \mathrm{t} \mathrm{u} \mathrm{d} \\
\text { retrospectivo }\end{array}$ & $\begin{array}{l}71,5 \% \text { receberam } \\
\text { e x t r a t o s } \\
\text { balanceados de } \\
\text { CBD:THC; } 19,0 \% \text { de } \\
\text { alto CBD; e 9,5\% de } \\
\text { extratos } \\
\text { com alto teor de } \\
\text { THC }\end{array}$ & $\begin{array}{l}\text { Melhora em pelo menos } \\
\text { um dos principais sintomas } \\
\text { do TEA. }\end{array}$ & (40) \\
\hline$N=20$ & $\begin{array}{l}\text { Crianças com TEA } \\
\text { (Estados Unidos) }\end{array}$ & $\begin{array}{l}\text { E } s \text { t u d o } \\
\text { retrospectivo }\end{array}$ & Cannabis medicinal & $\begin{array}{l}\text { Melhora nos sintomas de } \\
\text { humor, na frequência e } \\
\text { gravidade das crises, no } \\
\text { sono, humor, habilidades } \\
\text { de comunicação e } \\
\text { concentração. }\end{array}$ & 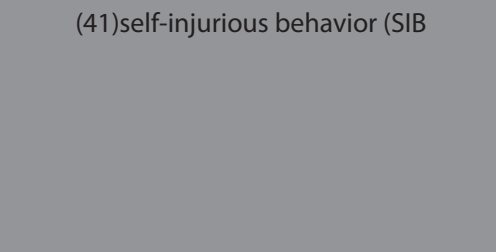 \\
\hline$N=1$ & $\begin{array}{l}\text { Criança com TEA e } \\
\text { epilepsia (Canadá) }\end{array}$ & Relato de caso & $\begin{array}{l}\text { Extrato a base de } \\
\text { canabidiol }(1: 20- \\
\text { THC } 0,001 \% \text { e CBD } \\
0,02 \%)\end{array}$ & $\begin{array}{l}\text { Melhora nos sintomas } \\
\text { comportamentais e sociais } \\
\text { essenciais relacionados ao } \\
\text { TEA, bem como ansiedade, } \\
\text { distúrbios do sono e peso. }\end{array}$ & (38) \\
\hline
\end{tabular}

Legenda: $T E A=$ Transtorno do espectro autista; $P G C=$ Problema grave de concentração; $T H C=$ Tetrahidrocanabinol; $C B D=C a n a b i d i o l ;$ TDAH=Transtorno do déficit de atenção com hiperatividade. 
10. Fleury-Teixeira P, Caixeta FV, da Silva LCR, Brasil-Neto JP, Malcher-Lopes R. Effects of cbd-enriched cannabis sativa extract on autism spectrum disorder symptoms: An observational study of 18 participants undergoing compassionate use. Front Neurol. 2019;10(OCT):1-9.

11. Baio J, Wiggins L, Christensen DL, Maenner MJ, Daniels J, Warren Z, et al. Prevalence of Autism Spectrum Disorder Among Children Aged 8 Years - Autism and Developmental Disabilities Monitoring Network, 11 Sites, United States, 2014. MMWR Surveill Summ [Internet]. 2018 Apr 27;67(6):1-23.

12. Sena T. Manual Diagnóstico e Estatístico de Transtornos Mentais - DSM-5, estatísticas e ciências humanas: inflexões sobre normalizações e normatizações. Vol. 11, Revista Internacional Interdisciplinar INTERthesis. 2014. 96 p.

13. Poleg S, Golubchik P, Offen D, Weizman A. Cannabidiol as a suggested candidate for treatment of autism spectrum disorder. Prog Neuro-Psychopharmacology Biol Psychiatry. 2019;89:90-6.

14. Araújo L. Manual de Orientação Transtorno do Espectro do Autismo. Soc Bras Pediatr. 2019;00:1-24.

15. Carter MT, Scherer SW. Autism spectrum disorder in the genetics clinic: A review. Clin Genet. 2013;83(5):399-407.

16. Griesi-Oliveira K, Sertié AL. Autism spectrum disorders: an updated guide for genetic counseling. Einstein (Sao Paulo). 2017;15(2):233-8.

17. Ronald A, Hoekstra RA. Autism spectrum disorders and autistic traits: A decade of new twin studies. Am J Med Genet Part B Neuropsychiatr Genet. 2011;156(3):255-74.

18. Gyawali S, Patra BN. Autism spectrum disorder: Trends in research exploring etiopathogenesis. Psychiatry Clin Neurosci. 2019;73(8):466-75.

19. Siu MT, Weksberg R. Epigenetics of autism spectrum disorder. Adv Exp Med Biol. 2017;978:63-90.

20. Leite R, Meirelles L, Milhomem D. Medicamentos usados no tratamento psicoterapêutico de crianças autistas em Teresina - PI. Drugs used in the psychotherapeutic treatment of autistic children in. Bol Inf Geum. 2015;6(3):91-7.

21. Vismara LA, Rogers SJ, Ogletree BT, Oren T, Mcphilemy C, Dillenburger K, et al. Havioral. J Autism Dev Disord. 2010;40(1):18-41.

22. Nikolov R, Jonker J, Scahill L. Autismo: Tratamentos psicofarmacológicos e áreas de interesse para desenvolvimentos futuros. Rev Bras Psiquiatr. 2006;28(SUPPL.

\section{1):39-46.}

23. Brenneisen R. Chemistry and Analysis of Phytocannabinoids and Other Cannabis Constituents. Marijuana and the Cannabinoids. 2007;(7):17-49.

24. Gu B. Cannabidiol provides viable treatment opportunity for multiple neurological pathologies of autism spectrum disorder. Glob Drugs Ther. 2017;2(6):1-4.

25. Devinsky O, Cilio MR, Cross H, Fernandez-Ruiz J, French J, Hill C, et al. Epilepsy and Other Neuropsychiatric Disorders. Epilepsia. 2014;55(6):791-802.

26. Cheng D, Low JK, Logge W, Garner B, Karl T. Chronic cannabidiol treatment improves social and object recognition in double transgenic APPswe/PS1 $\triangle$ E9 mice. Psychopharmacology (Berl). 2014;231(15):3009-17.

27. Zamberletti E, Gabaglio M, Parolaro D. The endocannabinoid system and autism spectrum disorders: Insights from animal models. Int J Mol Sci. 2017;18(9):1-14.

28. Masi A, DeMayo MM, Glozier N, Guastella AJ. An Overview of Autism Spectrum Disorder, Heterogeneity and Treatment Options. Neurosci Bull. 2017;33(2):183-93.

29. Karhson DS, Hardan AY, Parker KJ. Endocannabinoid signaling in social functioning: An RDoC perspective. Transl Psychiatry [Internet]. 2016;6(9):e905-8.

30. Englund A, Morrison PD, Nottage J, Hague D, Kane F, Bonaccorso $\mathrm{S}$, et al. Cannabidiol inhibits THC-elicited paranoid symptoms and hippocampal-dependent memory impairment. J Psychopharmacol. 2013 Jan;27(1):19-27.

31. Schubart CD, Sommer IEC, van Gastel WA, Goetgebuer RL, Kahn RS, Boks MPM. Cannabis with high cannabidiol content is associated with fewer psychotic experiences. Schizophr Res. 2011 Aug;130(1-3):216-21.

32. García C, Palomo-Garo C, García-Arencibia M, Ramos J, Pertwee R, Fernández-Ruiz J. Symptom-relieving and neuroprotective effects of the phytocannabinoid $\triangle^{9}$-THCV in animal models of Parkinson's disease. $\mathrm{Br} J$ Pharmacol. 2011 Aug;163(7):1495-506.

33. Anderson CL, Evans VF, Demarse TB, Febo M, Johnson CR, Carney PR. Cannabidiol for the Treatment of Drug-Resistant Epilepsy in Children: Current State of Research. J Pediatr Neurol. 2017;15(4):143-50.

34. Bar-Lev Schleider L, Mechoulam R, Saban N, Meiri G, Novack V. Real life Experience of Medical Cannabis Treatment in Autism: Analysis of Safety and Efficacy. Sci Rep [Internet]. 2019;9(1):1-7.

35. Franco GRR, Viegas $C$. The contribution of studies with canabidiol and synthetic analogues in the design of 
new drug candidates for neuropsychiatric disorders and neurodegenerative diseases. Rev Virtual Quim. 2017;9(4):1773-98.

36. Matos RLA, Spinola LA, Barboza LL, Garcia DR, França TCC, Affonsoa RS. The cannabidiol use in the treatment of epilepsy. Rev Virtual Quim. 2017;9(2):786-814.

37. Kurz R, Blaas K. Use of dronabinol (delta-9-THC) in autism : A prospective single-case-study with an early infantile autistic child. Cannabinoids. 2010;5(4):4-6.

38. Ponton JA, Smyth $K$, Soumbasis $E$, Llanos SA, Lewis $M$, Meerholz WA, et al. A pediatric patient with autism spectrum disorder and epilepsy using cannabinoid extracts as complementary therapy: A case report. J Med Case Rep. 2020;14(1):1-7.

39. Barchel D, Stolar O, De-Haan T, Ziv-Baran T, Saban N, Fuchs DO, et al. Oral cannabidiol use in children with autism spectrum disorder to treat related symptoms and Co-morbidities. Front Pharmacol. 2019;9(JAN):1-5.

40. Kuester G, Vergara K, Ahumada A, Gazmuri AM. Oral cannabis extracts as a promising treatment for the core symptoms of autism spectrum disorder: Preliminary experience in Chilean patients. J Neurol Sci. 2017;381:932-3.

41. McVige J, Headd V, Alwahaidy M, Lis D, Kaur D, Albert $B$, et al. Medical Cannabis in the Treatment of Patients with Autism Spectrum Disorder (1648). Neurology. 2020;14:94(15 Supplement):1648. 